\title{
A Surgical Technique for Congenital Preauricular Sinus
}

Heon Yoo,

Dong Ha Park,

II Jae Lee, Myong Chul Park

Department of Plastic and Reconstructive Surgery, Ajou University Hospital, Ajou University School of Medicine, Suwon, Korea
No potential conflict of interest relevant to this article was reported.

\begin{abstract}
Background: Preauricular sinuses represent a common congenital abnormality in children. Classically, a preauricular sinus manifests as a small opening, usually near the anterior limb of ascending helix. The difficulty in the surgical treatment of preauricular sinus is the high recurrence rate. The aim of this article is to review the outcomes of preauricular sinus and to introduce our surgical technique and its prognosis.

Methods: A single-institutional retrospective review was performed for all patients who had undergone excision of congenital periauricular sinus between October 2007 and April 2014. Medical records were reviewed for demographic information, wound complication, and recurrence rate. The sinus tract was visualized with the aid of preoperative dye instillation and intraoperative probe insertion. The skin next to the sinus opening was incised elliptically, and the tract itself was dissected medially to the end of the sinus tract and posteriorly to the cartilage of the ascending helix.

Results: The review identified 44 patients for a total of 57 preauricular sinus tracts. The mean age at time of operation was 16.3 years with a range from 9 months to 65 years. Unilateral preauricular sinus tract was present in 31 patients (11 right and 20 left preauricular tract), and 13 patients had bilateral sinus tract. None of the patients had experienced wound issues postoperative, and there were no recurrent sinus tract formation or infection. Conclusion: Using a combination of dye instillation, probe insertion, and modified dissection, we were able to achieve a recurrence free series of preauricular sinus tract excision among a heterogenous group of patients. A large patient series is necessary to replicate the results of this study.
\end{abstract}

Keywords: Preauricular fistulae / Surgical procedures / Follow-up / Prognosis

\section{INTRODUCTION}

First described by Heusinger in 1865, preauricular sinus is a common congenital abnormality in childhood. The incidence ranges from $0.1 \%$ to $0.9 \%$ in the Caucasian populations, although higher incidences have been reported in those of African ancestry and in East Asian populations ( $4 \%$ and 10\%, respectively) [1]. More specifically, they are found with an estimated incidence of $0.1 \%-0.9 \%$ in the United States and Europe, $2.5 \%$ in Taiwan, $4 \%-10 \%$ in some

Correspondence: Myong Chul Park

Department of Plastic and Reconstructive Surgery, Ajou University Hospital, 206

World cup-ro, Yeongtong-gu, Suwon 16499, Korea

E-mail: mpark@ajou.ac.kr

Received June 25, 2015 / Revised July 16, 2015 / Accepted August 5, 2015
African countries, and 2.53\% in Korea [2].

Most people with this malformation are asymptomatic. Some people, however, may present facial cellulitis or ulcerations located on the anterior side, contiguous to the ear [3]. Although there is no necessity to treat patients with no clinical signs or symptoms, improper management of patients with infected sinus will result in recurrent infection and possibly severe postoperative scarring [4].

In the acute phase of preauricular sinus infection, clinical management hinges on appropriate antibiotic therapy directed against the causative pathogen. Abscesses should be drained. Recurrent or persistent infection of preauricular sinus requires surgical excision of the sinus and its tract during the quiescent portion of the recurrent inflammatory cycle [5]. 
In 1990, Prasad et al. [6] first reported a supra-auricular approach with a lower recurrence rate of 5\%. In 2001, Lam et al. [7] reported that the standard technique and supra-auricular approach had recurrence rates of $32 \%$ and $3.7 \%$, respectively. In this study, we aim to review the surgical methods and outcomes in the management of preauricular sinus and present a simple and modified surgical technique that most surgeons can follow without difficulty.

\section{METHODS}

A retrospective review was performed for all patients undergoing surgical excision of preauricular sinus at a tertiary hospital between October 2007 and April 2014. Medical records were reviewed for demographic information, operative indication, operative findings, and postoperative outcomes. Long-term outcomes were evaluated via telephone interview.

Patients were managed in the following manner. An acutely infected preauricular sinus was first treated with a course of antibiotics. Any abscess was incised and drained as close to the sinus pit to facilitate future excision, and operation was delayed until signs of inflammation subsided.

One day prior to excisional operation, the sinus tract was filled with gentian violet solution via a polyethylene cap of 24-gauge intravenous catheter (Fig. 1). After general or local anesthesia, the skin around sinus opening was incised in an ellipse. A probe was inserted into the sinus tract to a depth permitting gentle resistance. The tract was dissected away from the surrounding subcutaneous fat, while evaluating tract wall injury by visual inspection of gentian violet blue dye. The tract was followed medially and posteriorly to the cartilage of the ascending helix, as in the supra-auricular approach. After examining for possible sinus branches, the tract was excised en bloc along with the attached portion of the ear cartilage (Fig. 2). Upon hemostasis, the wound was closed in layers.

\section{RESULTS}

The review identified 57 cases of preauricular sinus tract amongst 44 patients. There was a predilection for female patients (26 female to 18 male). The mean age at time of operation was 16.3 years with a range from 9 months to 65 years. Unilateral preauricular sinus tract was present in 31 patients (11 right and 20 left preauricular tract), and 13 patients had bilateral sinus tract (Table 1). In 33 cases, the operations were performed under the general anesthesia and in 11 cases under local anesthesia.

The most common indication for sinectomy was chronic malordorous discharge at the time of presentation (35.1\%), fol-

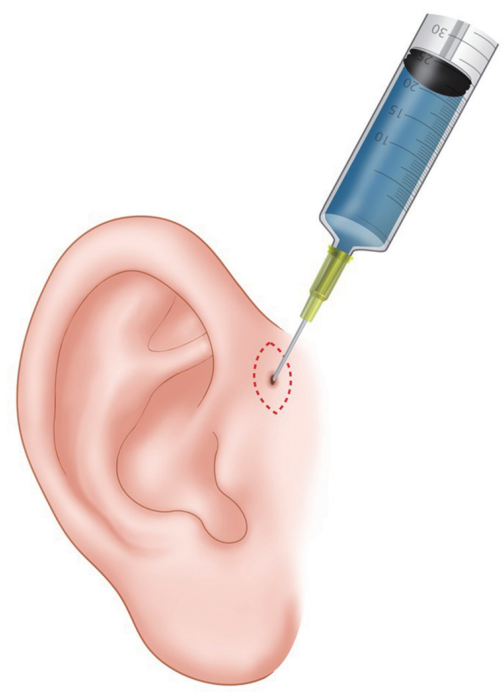

Fig. 1. G-V solution injection to preauricular sinus with a polyethylene cap of 24-gauge intravenous catheter on the day prior to surgery.

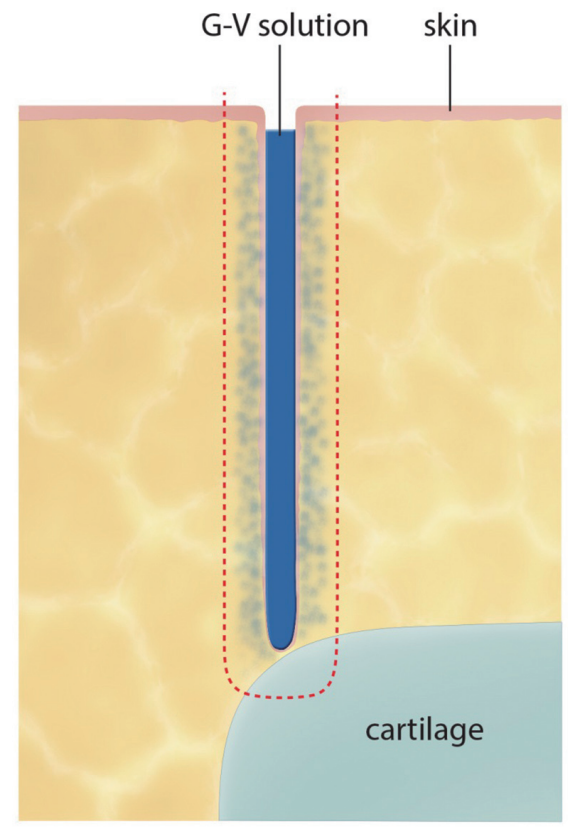

Fig. 2. Schematic diagram of our surgical technique for preauricular sinus. 
lowed by asymptomatic patients simply wishing to have the tract removed for either psychologic or cosmetic reasons (31.6\%). Patients who presented with prior history of infection consisted of $22.8 \%$ of patients. The presence of chronic discharge only (10.5\%) was less frequent (Table 2).

Patient did not experience any anesthesia or perioperative complications. All of the surgical sites had healed well without wound dehiscence or surgical site infection. The mean follow up period from the final operation was 56 months (range, 15-95 months), and none of the patients had experienced recurrent sinus tract formation (Table 3). Out of the 44 patients, 36 patients were

Table 1. Patient characteristics $(n=44)$

\begin{tabular}{|c|c|}
\hline Characteristic & Value \\
\hline \multicolumn{2}{|l|}{ Sex, n (\%) } \\
\hline Male & $18(41)$ \\
\hline Female & $26(59)$ \\
\hline \multicolumn{2}{|l|}{ Age, yr } \\
\hline Oldest & 65 \\
\hline Youngest & 0.75 \\
\hline Mean & 16.4 \\
\hline \multicolumn{2}{|l|}{ Location, n (\%) } \\
\hline Right & $11(25)$ \\
\hline Left & $20(45)$ \\
\hline Bilateral & $13(30)$ \\
\hline
\end{tabular}

Table 2. Indication for operation $(\mathrm{n}=57)$

\begin{tabular}{lc}
\hline Symptoms & Frequency \\
\hline Both of discharge and odor & $20(35.1)$ \\
\hline No symptom & $18(31.6)$ \\
\hline Previous infection(s) & $13(22.8)$ \\
\hline Chronic discharge & $6(10.5)$ \\
\hline Odor & $0(0)$ \\
Recurrence & $0(0)$ \\
\hline
\end{tabular}

Values are presented as number (\%).

Table 3. Follow-up period and complications after operation

\begin{tabular}{lc} 
Contents & Value \\
\hline Follow up period (mean), mo & 15-95 (56) \\
\hline Complication, $\mathrm{n}(\%)$ & \\
\hline Wound dehiscence & 0 \\
Wound infection & 0 \\
\hline Recurrence & 0 \\
\hline
\end{tabular}

available for telephone interview, among whom two patients reported pruritus at the surgical site.

\section{DISCUSSION}

The external ear is formed embryologically from six hillocks of the first and second branchial arch. The formation of preauricular sinus results from failure of complete fusion of these mesenchymal mounds. The preauricular sinus, therefore, is closely related to the groove between the tragus and cartilage of the anterior helix [8]. Other rare locations reported in the literature include superior to the ascending limb of the helix, along the posterior surface of the helical crus, cymba concha, ear lobule, and postauricular area [1]. In literature, the incidence of variant-type preauricular sinus is unknown, and only two studies have reported greater than 10 cases of unusual preauricular sinus tract prior to 2014 [2].

The classical approach to surgical excision of these tracts are associated with a high recurrence rate, ranging from $22 \%$ to $42 \%$. This is most likely due multiple branches of the sinus tracts which are frequently missed during the excision [8]. In this context, the single most important factor influencing surgical outcome following sinectomy is whether the surgeon has the means to identify, isolate, and excise every single terminal branches of the sinus. All of this is made more difficult in patients with prior history of infection, which often lead to fibrosis that alters the sinus route [7]. This is the reason behind the wide spectrum of techniques available for preauricular sinus tracts.

Most of the variations in surgical technique are developed with the goal of reducing the recurrence rate. In a review of the English literature, Gan et al. summarized 11 studies concerning surgical outcomes following preauricular sinectomy. The authors found that adequate sinus tract visualization is an important factor in reducing recurrence rates. Most of the technical variations in that review included a combination of methylene blue dye instillation, tract probe, and/or the use of microscope, and such techniques were associated with recurrence rates in between 0 and $2 \%[1]$.

In addition to the methods to improve the visualization of sinus tract branches, the supra-auricular approach has been received favorably amongst surgeons [1]. This technique is based on the iden- 
tification of temporalis fascia as the medial border of dissection and of the cartilage of helix and auditory canal as the posterior border of dissection. This approach allows en-bloc resection of the sinus tree and is associated with recurrence rates less than 5\% [8].

Nevertheless, the large dead space resulting from the original supra-auricular approach often necessitates insertion of a drain and postoperative compression dressing. Moreover, a large dead space in the wound may increase the risk of postoperative infection and hematoma [9]. Furthermore, the approach also results in a lengthy scar. While the variations in surgical methods are associated with lower recurrence rates [2], unfamiliarity with the preauricular sinus and/or limited experience with the variations in surgical techniques should also be considered.

At our institution, preauricular sinus tracts are visualized and controlled with preoperative dye instillation and intraoperative probe exploration. Dye instillation one day prior to surgery and excision of adjacent cartilage to sinus is a wonderful method for visualizing the tract. If injected a day prior to operation, the dye will have enough time to spread from the main tract into the finer branches.

We do not perform as extensive of a dissection as in the supraauricular approach to avoid the problem with dead space and lengthy scar. Excision of cartilage adjacent to the sinus tract is also helpful in further decreasing the possibility of remnant tract (Fig. 3).

There are a few limitations in our study. The retrospective study

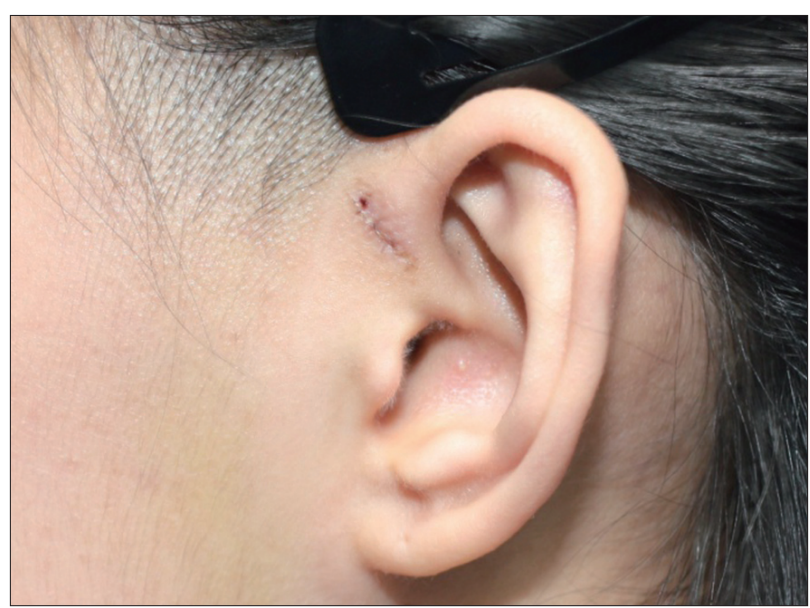

Fig. 3. Photograph of a 15 -year-old patient on postoperative day 7. contained a small number of cases, and we are not able to eliminate the possibility of selection and confirmation bias. However, information on recurrences and complications were updated by phone interview at least one year after surgery, which is not a short period, and the results of our research were the expected effectiveness with no simple case of recurrence in follow up.

Many surgical methods have been described for complete excision of congenital perauricular sinus. The problem in the surgical removal of preauricular sinus is the recurrence. It is result from the high variability of the sinus ramifications, and terminal ramifications is difficult to be confirmed. We suggest that our technique could provide a tool for verifying terminal site of sinus. With shorter incisional scars, the tenique described in this article could be a preferred option in surgical treatment for congenital preauricular sinus.

\section{REFERENCES}

1. Gan EC, Anicete R, Tan HK, Balakrishnan A. Preauricular sinuses in the pediatric population: techniques and recurrence rates. Int J Pediatr Otorhinolaryngol 2013;77:372-8.

2. Kim JR, Kim do H, Kong SK, Gu PM, Hong TU, Kim BJ, et al. Congenital periauricular fistulas: possible variants of the preauricular sinus. Int J Pediatr Otorhinolaryngol 2014;78:1843-8.

3. Yeo SW, Jun BC, Park SN, Lee JH, Song CE, Chang KH, et al. The preauricular sinus: factors contributing to recurrence after surgery. Am J Otolaryngol 2006;27:396-400.

4. Shim HS, Kim DJ, Kim MC, Lim JS, Han KT. Early one-stage surgical treatment of infected preauricular sinus. Eur Arch Otorhinolaryngol 2013;270:3127-31.

5. Tan T, Constantinides H, Mitchell TE. The preauricular sinus: a review of its aetiology, clinical presentation and management. Int J Pediatr Otorhinolaryngol 2005;69:1469-74.

6. Prasad S, Grundfast K, Milmoe G. Management of congenital preauricular pit and sinus tract in children. Laryngoscope 1990;100:320-1.

7. Lam HC, Soo G, Wormald PJ, Van Hasselt CA. Excision of the preauricular sinus: a comparison of two surgical techniques. Laryngoscope 2001;111:317-9.

8. Baatenburg de Jong RJ. A new surgical technique for treatment of preauricular sinus. Surgery 2005;137:567-70.

9. Bae SC, Yun SH, Park KH, Chang KH, Lee DH, Jeon EJ, et al. Preauricular sinus: advantage of the drainless minimal supra-auricular approach. Am J Otolaryngol 2012;33:427-31. 